\title{
Potenciar las competencias soft skills con presentación oral en el aula
}

\section{Gaona, Cristina ${ }^{a}$}

${ }^{a}$ Dpto. Economía y Ciencias Sociales. Universitat Politècnica de València (UPV) Camino de Vera, s/n 46022 Valencia. España. crigaoga@esp.upv.es

\begin{abstract}
The current labor complexity requires not only technical knowledge, but also soft skills - among the most outstanding are teamwork and leadership (CT06), effective communication (CT-08), planning and time management (CT12). The challenge of soft skills is to move from theoretical knowledge to practice with active methodologies after the pressure of the Bologna Process. The goal of this experimental session is to increase the soft skills of the students with a dynamic session of two hours through an oral presentation that improves the participation and motivation of the students. The session has five parts: instructions and creation of teams, preparation, oral presentation and feedback between peers. The good results in the improved skills, the satisfaction and the recommendation of the students allow to extend this dynamic to other groups.
\end{abstract}

Keywords: experiential learning activity, soft skills, transverse skills, competences, active methodology, agile methodology, oral presentation.

\section{Resumen}

La complejidad laboral actual requiere no solo conocimientos técnicos, sino también de habilidades soft skills - entre las más destacadas se encuentran: el trabajo en equipo y el liderazgo (CT-06), la comunicación efectiva (CT08), la planificación y la gestión de tiempo (CT-12). El desafio de las soft skills está en pasar de los conocimientos teóricos a su práctica con metodologías activas tras la presión del Proceso Bolonia. El objetivo de esta sesión experimental es incrementar las soft skills de los estudiantes con una sesión dinámica de dos horas a través de una exposición oral que mejore la participación y motivación de los estudiantes. La sesión tiene cinco partes: instrucciones y creación de los equipos, preparación, exposición oral y retroalimentación entre pares. Los buenos resultados en las habilidades soft skills mejoradas, la satisfacción y la recomendación de los estudiantes permite extender esta dinámica a otros grupos.

Palabras clave: aprendizaje experiencial, competencias transversales, competencias esenciales, competencias, metodología activa, metodología ágil, exposición oral. 


\section{Introducción}

En las universidades han primado tradicionalmente las hard skills o habilidades técnicas frente a las soft skills o habilidades esenciales. Actualmente, las soft skills son consideradas un predictor de éxito profesional y la sociedad empresarial sigue insistiendo en la existencia de un gap histórico. En 1918, un estudio publicado por la Fundación Carnegie indicaba la necesidad de potenciar estas habilidades en la educación universitaria de ingenieros (Mann, 1918). En 2019, Linked In recoge en su Informe anual sobre las Tendencias de Contratación de Talento (encuestados 5.000 Directivos RRHH) las soft skills como una de las cuatro tendencias más destacadas, entre estas destacan: liderazgo, trabajo colaborativo en equipo, comunicación y gestión del tiempo. En esta investigación el 92\% de los directivos afirmó que las soft skills son más importantes que las habilidades técnicas, además el 89\% indicaron que las malas contrataciones vienen determinadas por la falta de soft skills (Linked In, 2019).

La implantación del Espacio Europeo de Educación Superior (EEES) incrementó las horas de prácticas que permiten mejorar las competencias en soft skills o competencias transversales (CT), pero también se incrementaron las expectativas sobre estas por parte de los estudiantes, puesto que éstos conocen que su futura empleabilidad depende de estas habilidades (Bruneel, D’Este, \& Salter, 2010). Las soft skills son las habilidades relacionadas con el desarrollo personal, que no dependen de un ámbito temático o disciplinario específico, sino que aparecen en todos los dominios de la actuación profesional y académica (González \& Wagenaar, 2003).

Las universidades están escuchando las demandas empresariales respecto a una necesidad de mayor formación en soft skills, puesto que los conocimientos técnicos no son suficientes para el desarrollo profesional (Goswami, 2013). Sin embargo, la innovación docente y la incorporación de nuevos contenidos en los grados y postgrados se ven limitados por los procesos de acreditación y verificación y el tiempo limitado del calendario docente (ECTS) (Prado, 2012). Con la entrada del Proceso Bolonia, las estructuras de los estudios cambiaron las licenciaturas (5 años) a grados (4 años) y ahora se planifica el " $3+2$ ” que permita mejorar el Espacio Europeo de Educación Superior. Estas transformaciones educativas han reducido la autonomía de las universidades y la libertad de cátedra de los docentes (Prado, 2012). Por el contrario, el Proceso Bolonia ha impulsado un cambio en las metodologías docentes para que el estudiante "aprenda a aprender haciendo".

El desafío de las soft skills está en pasar de los conocimientos teóricos a su práctica. Para ello son necesarias las metodologías activas que ponen al estudiante como protagonista de su aprendizaje. Se entiende como metodologías activas aquellos métodos y estrategias que utiliza el docente para convertir el proceso de enseñanza en actividades que fomenten la participación activa del estudiante y lleven al aprendizaje (Labrador \& Andreu, 2008). Estas metodologías deben proponer una gradación coherente de la Taxonomía de Bloom y poner las prácticas en el centro del aprendizaje, siguiendo la pirámide de aprendizaje de 
Cody Blair. Algunas de estas metodologías son: la clase inversa, el aprendizaje basado en problemas, aprendizaje por proyectos (PBL), gamificación, simulación, método del caso, metodologías ágiles, etc. (Labrador \& Andreu, 2008).

\section{Objetivos}

El objetivo prioritario de esta propuesta de innovación docente es utilizar la presentación oral para potenciar las soft skills destacadas en el Informe Anual de Contratación de Talento (Linked In, 2019), que tienen su equivalente en las siguientes competencias transversales de la Universidad Politécnica de Valencia (tabla 1).

\section{Tabla 1. Equivalencia soft skills Linked In con Competencias Transversales UPV}

\begin{tabular}{|ll|}
\hline Competencias soft skills (Linked in) & Competencias Transversales de la UPV \\
Liderazgo & CT-06 Trabajo en equipo y liderazgo \\
Trabajo colaborativo en equipo & CT-06 Trabajo en equipo y liderazgo \\
Comunicación & CT-08 Comunicación efectiva \\
Gestión del tiempo & CT-12 Planificación y gestión del tiempo \\
\hline
\end{tabular}

Fuente: Linked in (2019) y UPV (2016)

Otros objetivos de esta innovación docente son:

- Diseñar una dinámica de aprendizaje experiencial activo.

- Incrementar la motivación y participación del estudiante.

- Producir material educativo para soportar esta metodología en el aula.

- Acercar casos de éxito empresarial y visualizar el contexto empresarial global.

- Potenciar tres competencias transversales (CT-06, CT-08, CT-12).

- Analizar la percepción de los estudiantes respecto a la innovación docente.

- Valorar los resultados obtenidos en las soft skills de los estudiantes.

\section{Metodología}

Las metodologías ágiles pretenden resolver problemas complejos de forma flexible y en periodos cortos de tiempo para lograr un proceso rápido. Además, la capacidad para transmitir ideas de forma ágil, clara y sencilla, de una manera convincente e incluso, atrayente, se ha convertido en un elemento capital (San Tan \& Ng, 2006), puesto que estamos en un contexto VUKA (Volátil, Incierto, Complejo y Ambiguo) y de creciente digitalización. Las metodologías ágiles vienen del concepto originario de "desarrollo ágil" para favorecer las interacciones en el desarrollo de software (Beck, et al., 2001). La idea principal de una metodología ágil es reducir el coste del intercambio de información del equipo y reducir el tiempo en la toma de decisión para conseguir resultados exitosos (Cockburn, 2001). Todas las metodologías ágiles deben ser claras, dinámicas, adaptativas $\mathrm{y}$ flexibles para abordar cualquier problema potencial, y la comunicación eficiente y efectiva entre el equipo se considera fundamental para el éxito de cualquier proyecto. 
El diseño de esta sesión se basa en la metodología ágil para realizar un proyecto concreto, en esta ocasión mediante una presentación oral que responde a una serie de cuestiones y sigue unos criterios claros y concisos (Romero et al., 2017):

- Contenido: debe responder a 3 preguntas (basadas en la técnica de las $5 \mathrm{~W}$ y $1 \mathrm{H}$ )

- ¿Qué es la empresa X?,

- ¿Cuál es su público objetivo prioritario?

- ¿Qué solucionan sus servicios?

- Estructura: coherencia, cohesión y concreción.

- Exposición oral: claridad, pronunciación, ritmo y entonación

- Comunicación no verbal: lenguaje no verbal, postura, autocontrol y seguridad.

\section{Estructura de la sesión}

La sesión tiene una duración de dos horas en una sesión de prácticas de la asignatura de Dirección Comercial en el doble grado de Administración y Dirección de Empresas + Ingeniería de las Tecnologías y Servicios de Telecomunicación de la Universidad Politécnica de Valencia. Es una asignatura obligatoria en cuarto curso. Toda la sesión se desarrolla en el aula, sin ninguna instrucción e información anterior sobre la misma. La sesión se divide en cinco partes (tabla 2):

Tabla 2. Partes y duración de la sesión

\begin{tabular}{|ll|}
\hline \multicolumn{1}{|c|}{ Parte de la sesión } & \multicolumn{1}{c|}{ Duración } \\
\hline 1.- Instrucciones, información y constitución equipos & 5 minutos \\
2.-Preparación exposición oral & 15 minutos \\
$\begin{array}{l}\text { 3.-Exposición oral } \\
\text { 4.-Evaluación de la exposición oral }\end{array}$ & 5 minutos/equipo \\
5.-Retroalimentación por pares & 5 minutos/equipo \\
\hline
\end{tabular}

Fuente: elaboración propia.

1. Instrucciones, información y constitución de equipos. La docente forma aleatoriamente los equipos de trabajo con 4-5 miembros cada uno, designando un líder en cada uno de ellos. A continuación, se explican las instrucciones para llevar a cabo la presentación oral objeto de la sesión.

2. Preparación de la exposición oral. Los diferentes equipos responden a las preguntas, crean y ordenan las ideas para trasladarlas conjuntamente en la posterior exposición.

3. Exposición oral. Cada equipo responde a las preguntas realizadas a través de una exposición clara, concisa y equitativa por parte de todos los miembros del equipo.

4. Evaluación de la exposición oral por parte de los estudiantes oyentes. El resto de los equipos analizan según los criterios anteriormente indicados cada exposición oral.

5. Retroalimentación por pares. Cada equipo recibe esquemáticamente por escrito las mejoras y las felicitaciones correspondientes a su exposición. Además, tras la exposición se verbaliza por parte de cada equipo lo más destacado de la exposición recién realizada, con el objetivo de conseguir un aprendizaje continuo e incremental. 


\section{Competencias transversales}

El objetivo prioritario de la sesión es potenciar tres soft skills que se corresponden con tres competencias transversales (UPV): CT-06: Trabajo en equipo y liderazgo, CT-08: La comunicación efectiva, CT-12: La planificación y gestión de equipos. En cada parte de la sesión se trabajan distintas competencias transversales (tabla 3):

Tabla 3. Partes y competencias transversales que se trabajan en la sesión

\begin{tabular}{|ll|}
\hline \multicolumn{1}{|c}{ Partes de la sesión } & Competencias transversales que se trabajan \\
2.-Preparación exposición oral & CT-06: Trabajo en equipo y liderazgo \\
3.-Exposición oral & CT-08: La comunicación efectiva \\
& CT-12: La planificación y gestión de tiempos \\
4.-Evaluación de la exposición oral & CT-06: Trabajo en equipo y liderazgo \\
5.-Retroalimentación por pares & CT-08: La comunicación efectiva \\
& CT-12: La planificación y gestión de tiempos \\
\hline
\end{tabular}

Fuente: UPV (2016).

CT-06: Trabajo en equipo y liderazgo. Los estudiantes comparten conocimientos, responsabilidad y compromiso trabajando cooperativamente en equipo en toda la sesión.

CT-08: La comunicación efectiva. La exposición oral permite transmitir conocimientos y expresar ideas, así como en la retroalimentación por pares explicar las mejoras posibles.

CT-12: La planificación y gestión de tiempos. La importancia de organizarse y distribuir el tiempo, comprometiéndose y cumpliendo el objetivo en el límite temporal establecido.

\section{Resultados}

La sesión ha permitido asentar los contenidos específicos de la asignatura y potenciar las habilidades soft skills de los estudiantes: liderazgo, trabajo colaborativo en equipo, comunicación y gestión del tiempo, destacadas en el Informe de Linked In (2019).

Tabla 4. Competencias transversales mejoradas en la sesión (\%)

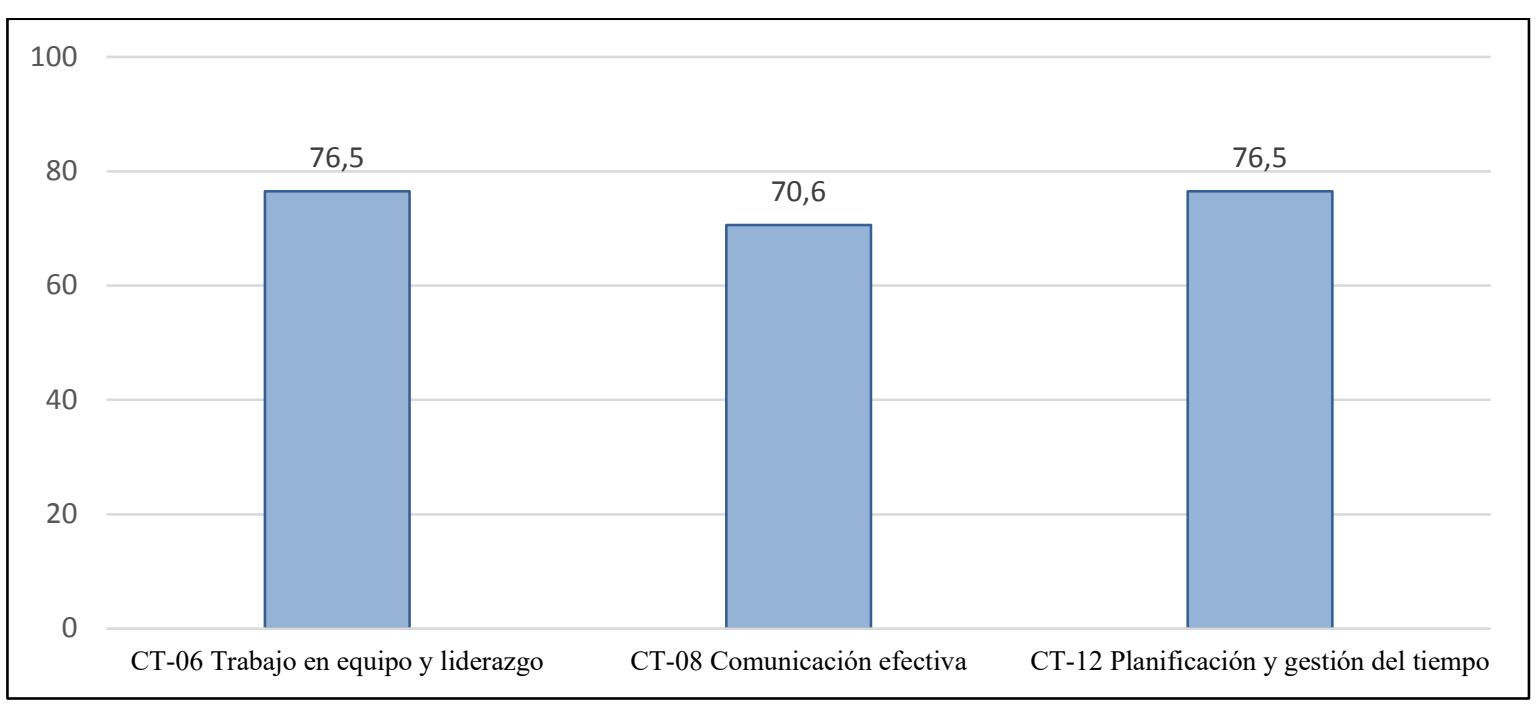

Fuente: elaboración propia 
La dinámica de aprendizaje experiencial activo ha incrementado la motivación y participación de los estudiantes y su conocimiento sobre casos de éxito empresariales actuales. Además, los resultados reflejan una alta satisfacción y una masiva recomendación que permiten extender esta dinámica a otros grupos y grados (tabla 5).

Tabla 5. Grado de satisfacción y recomendación de los estudiantes con la sesión

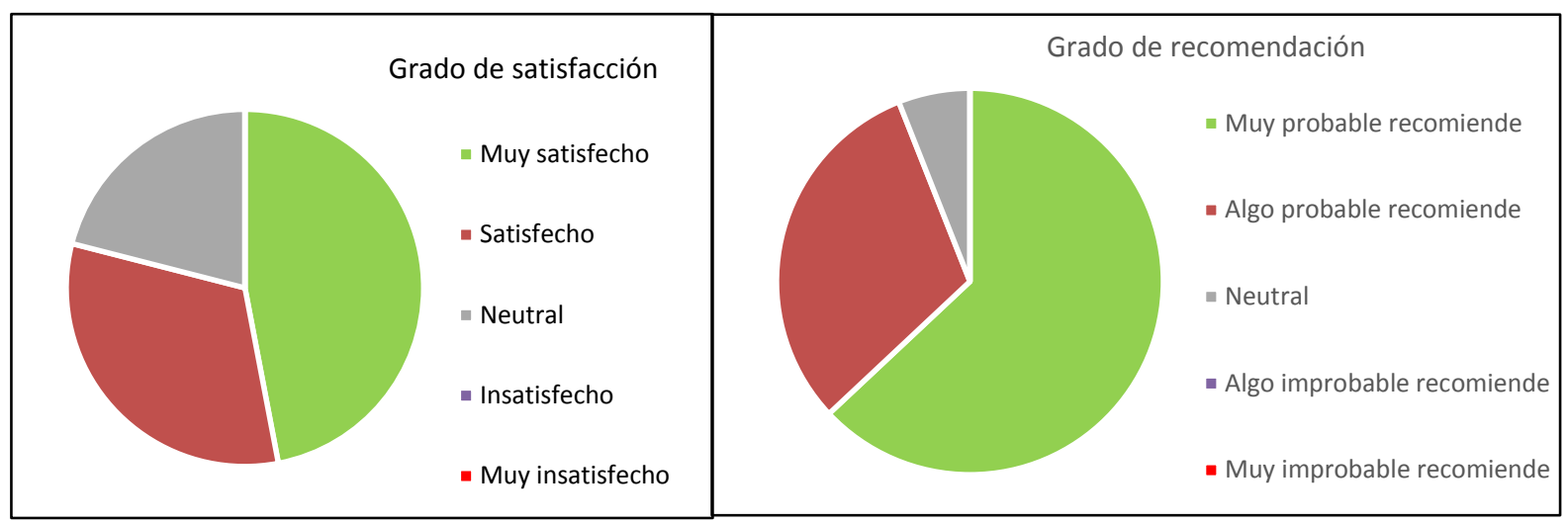

Fuente: elaboración propia.

\section{Conclusiones y recomendaciones}

La experiencia ha sido positiva por los buenos resultados sobre la mejora de las soft skills de los estudiantes, la satisfacción generalizada y la masiva recomendación de la sesión. Todo ello permite ampliar su uso a otros grupos y repetir este tipo de sesión ágil en otras sesiones prácticas incorporando otros contenidos. Esta dinámica ha conseguido la participación de los estudiantes durante las dos horas de duración y "han aprendido a aprender haciendo".

Las exposiciones orales han estado bien organizadas y expuestas. La mejora ha sido incremental entre cada uno de los equipos, gracias a la retroalimentación continua y de calidad entre pares. No se produce una discriminación negativa con la verbalización de las mejoras, puesto que la evaluación de esta practica no se traslada a la nota final.

Los estudiantes estuvieron motivados y comprometidos activamente en las distintas partes de la sesión. Una línea de mejora podría ser el uso de la "clase inversa" que permita adelantar conocimientos teóricos sobre la realización de las exposiciones orales, mejorando la preparación de la exposición oral y su puesta en escena.

El uso de multinacionales de éxito ha permitido, por su popularidad, la contextualización empresarial y la rápida interiorización de sus roles como directivos de éstas. Con el objetivo de incrementar los conocimientos empresariales en próximas sesiones se pueden incluir empresas locales de éxito que permitan potenciar sus capacidades emprendedoras al identificarse con los emprendedores locales. Para conseguirlo se anticipa telemáticamente una información básica de las empresas seleccionadas. 
Por último, la Universidad lleva trabajando las competencias transversales como prioridades en el Plan estratégico UPV 2015-2020. Una recomendación sería no sólo incrementar el trabajo sobre las competencias transversales en los grados y postgrados, sino como formación extracurricular específica en soft skills y complementar todo ello con concursos o challenges, dónde los estudiantes puedan ponerse a prueba y comprobar su nivel en soft skills.

\section{Referencias}

BAVIERA, T., BAVIERA PUIG, A., y BUITRAGO VERA, J. M. (2018). "Cómo evaluar la competencia transversal "trabajo en equipo" desde un enfoque $180^{\circ}$ en estudiantes universitarios". IV Congreso Nacional de Innovación Educativa y Docencia en Red (IN-RED 2018). Valencia: Universitat Politècnica València. en: $<$ https://doi.org/10.4995/INRED2018.2018.8733> y [Consulta: 15 junio de 2019]

BECK, K., ET AL. (2001). "Manifesto for Agile Software Development", 2001. $<$ http://scholar.google.co.in/scholar?q=agile+manifesto\&btnG $=\& h l=e n \& a s \_s d t=0,5 \&$ as ylo $=2009 \# 0>$ y [Consulta : 15 junio de 2019]

BRUNEEL, J., D'ESTE, P., y SALTER, A. (2010). Investigating the factors that diminish the barriers to university-industry collaboration. Research Policy, 39 (7), p. 858-868. $<$ https://doi.org/10.1016/j.respol.2010.03.006 y [Consulta : 15 junio de 2019]

COCKBURN, A. (2001). "Agile the people factor". Computer.Org, vol. November 131133. <https://www.computer.org/csdl/mags/co/2001/11/ry131.pdf y [Consulta : 15 junio de 2019]

GONZÁLEZ, J., y WAGENAAR, R. (2003). Tuning educational structures in Europe. $<$ http://www.ehea.info/media.ehea.info/file/Tuning_phase_II_2004/08/8/20040521_Tun ing_Invitation_579088.pdf y [Consulta : 15 junio de 2019]

GOSWAMI, R. (2013). Importance of soft skills in the employability of IT students. In National Conference on Emerging Trends: Innovations and Challenges in IT (pp. 19-20).

LABRADOR, M., y ANDREU, M. (2008). Metodologías activas. Valencia: Editorial Universitat Politècnica de València.

LINKED IN. (2019). "Global Talent Trends Report". Palo Alto: Editorial Linked In Disponible en: <https://business.linkedin.com/content/dam/me/business/en-us/talentsolutions/resources/pdfs/global_talent_trends_2019_emea.pdf y [Consulta: 15 junio de 2019]

MANN, C. R. (1918). A study of engineering education: prepared for the Joint Committee on Engineering Education of the National Engineering Societies. Editorial Merrymount Press.

PRADO, C. V. (2012). L'Espai Europeu d'Educació Superior i la seva implantació a les universitats espanyoles. Revista Catalana de Dret Públic, (44), p. 253-283.

ROMERO, E., ARTAL, J., ARTACHO, J., y ARANDA, J. G. (2017). "Aprendizaje invertido con Elevator Pitch y Pecha Kucha. In Aprendizaje invertido con Elevator Pitch y Pecha Kucha". V Congreso Internacional sobre Aprendizaje, Innovación y Competitividad (CINAIC 2017). Disponible en: <https://doi.org/DOI: 10.26754/CINAIC.2017.000001_139 y [Consulta: 15 junio de 2019]

SAN TAN, S., y NG, C. K. (2006). "A problem-based learning approach to entrepreneurship education". Education + Training, 48(6), p. 416-428. $<$ https://doi.org/10.1108/00400910610692606 y [Consulta : 15 junio de 2019] 